\title{
Experimental Study of Magnetic Fluid and Magnetic grease combination
}

\section{rotary Sealing*}

\author{
Feifei Xing ${ }^{1, a}$, Jun $\mathrm{Ji}^{2, b}$ \\ ${ }^{1}$ BMEI CO.,LTD., Beijing 100027, China \\ ${ }^{2}$ Beijing Polytechnic, Beijing 100176,China \\ axingfeifei@126.com, bji_jun2000@sina.com
}

Key words: Sealing; Magnetic fluid; Magnetic grease; Experiment

Abstract: The magnetic fluid and magnetic grease combination rotary Sealing structure device have been designed, and the experimental station and process of this structure was discussed. The results shows that,when the sealing gap were $0.3 \mathrm{~mm}, 0.4 \mathrm{~mm}, 0.5 \mathrm{~mm}$ and $0.6 \mathrm{~mm}$ respectively, the pressure capacity was mainly related with the sealing stage, magnetic field strength, magnetic fluid saturation magnetization and yield stress of magnetic grease.

\section{Introduction}

Magnetic fluid sealing is a new form of sealing method, it is rely on the liquid material to fill the gap to achieve sealing function. It has many advantages: zero leakage, long life, no pollution high reliability. It's typical sealing gap is $0.1 \mathrm{~mm}-0.3 \mathrm{~mm}^{[1]}$, but in many cases, in order to meet the high vibration resistance, the gap to be sealed structure is greater than $0.3 \mathrm{~mm}^{[2]}$. In these situation, use the traditional sealing method or other forms of sealing may have some difficulties: poor sealing functions, difficult to process the components ,difficult to assemble, complex structures and so on ${ }^{[4]}$. So it is gradually turned to study the magnetic fluid sealing which is a new sealing method, it is very important to study the large gap under the magnetic fluid sealing technology. The pressure performance,the leakage rate of magnetic fluid sealing and the life of the sealing structure are very important performance indicators ${ }^{[5]}$. This paper presents a magnetic fluid and magnetic grease combination rotary sealing structure, the gap can be adjusted from $0.3 \mathrm{~mm}$ to $0.7 \mathrm{~mm}$, it was also tested the pressure capacity and the leakage rate under the corresponding sealing gap. The results showed that the design of the combination sealing structure is not only can solve the large gap problems, but also improved the sealing pressure capability.

\section{The principle of magnetic fluid sealing}

In its typical form of the structure, a magnetic fluid seal (Fig.1)consists of two pole pieces, separated by an axially polarized permanent magnet, the magnetic fluid and the magnetically permeable shaft which is to be sealed. All these parts together form a closed magnetic circuit. The sharp-edged structures in the pole pieces (or in the shaft) locally build up a very small clearance between the dynamic (shaft) and static(pole piece). 


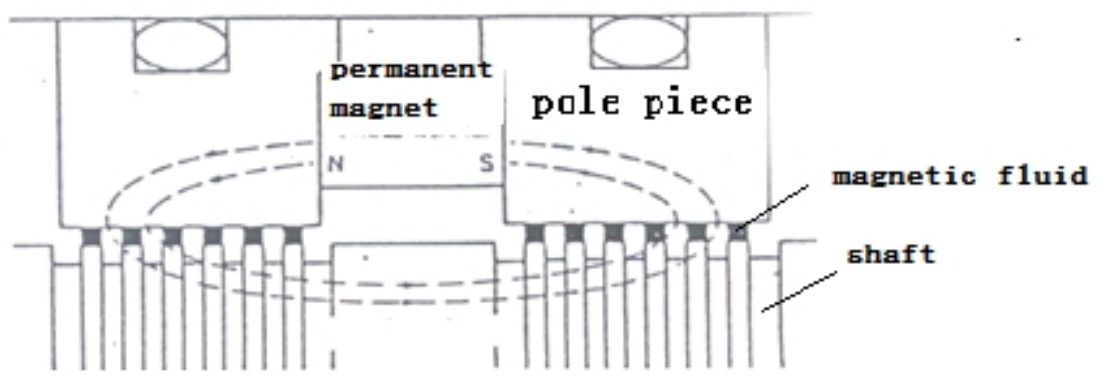

Fig.1 Principle diagram of typical magnetic fluid sealing structure Usually, the magnetic fluid seal's pressure resistance of each single-stage is ${ }^{[6]}$

$$
\Delta p \approx n \mu_{0} M_{s} \nabla H \approx n \mu_{0} M_{s}\left(H_{\max }-H_{\min }\right)
$$

Where the $\mu_{0}$ is the vacuum permeability, $M_{s}$ is the saturation magnetization of magnetic fluid, $\mathrm{n}$ is the number of pole piece, $\mathrm{H}$ is the magnetic field intensity in the seal gap, $H_{\max }$ and $H_{\min }$ is the maximum and the minimum of the magnetic field intensity within the magnetic fluid region.

By Formula (1), we can see that, the main factors of the magnetic fluid sealing capacity are sealing gap and the magnetic field distribution. However, the materials and the size of the magnet and the pole pieces are also determined the capacity, the main factor that decided the magnetic field distribution is the sealing gap. The experiment was to test the abilities of magnetic fluid sealing under different gaps.

\section{The experiments of magnetic fluid static sealing.}

The large gap magnetic fluid sealing structure was designed as figure 2 shows, it includes the sealing chamber, permanent magnet, the magnetic pole pieces and so on. There are five permanent magnets, each of them with its corresponding permanent magnet pole pieces can constitute more magnetic circuits, the teeth structures are the triangular shapes, the fisrt teeth is rectangular,and the number of the tooth is 9 , each teeth is the same size. The sealing chamber housing is 304 stainless steel non-magnetic materials, the permanent magnet material is $\mathrm{NdFeB}$, because it has large energy product, it can produced high strength magnetic field. The pole pieces are the iron, and the shaft is ICr13, we can get the different shaft sealing gaps by processing the center shaft.

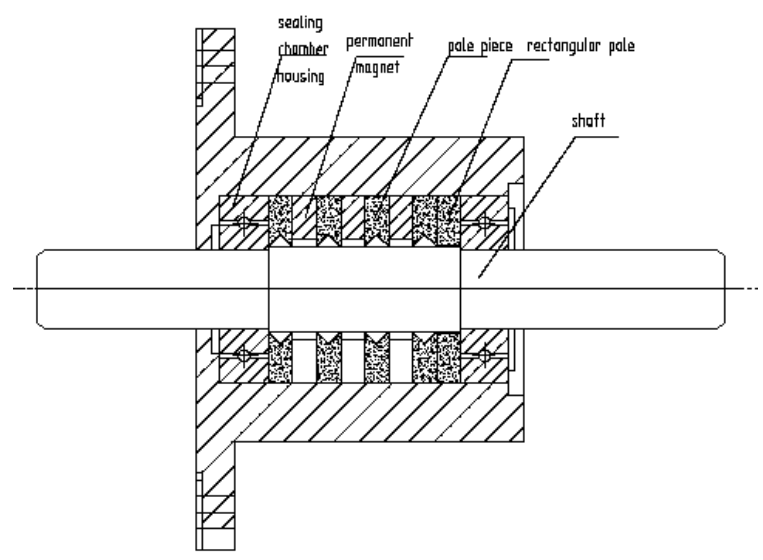

Fig.2 Device of magnetic fluid sealing 
The experiment was divided into two parts, the first part is to test the pressure capacity of the magnetic fluid seal, and the second is to test the leakage. In the first part, open the valve, making the high-pressure helium continuously fill the sealing chamber by regulating the valve. At the beginning, it was filled about 0.03 to $0.04 \mathrm{MPa}$ helium. Since then it is filled about $0.01 \mathrm{MPa}$ nitrogen every one or two minutes, at the same time it is determined whether the sealing damaged or not by helium leak detectors, and the barometer read the pressure when it is damaged. To get the different sealing gaps by processing the axis, and the data measured under different gaps show in Table 1. After 24 hours later, every magnetic fluid sealing was broken, recorded the pressure capacity, and because of the magnetic fluid's self-repair functions it can still be maintained to some pressure value. The data show in Table 1.

Tab.1 The relation between sealing gaps and anti-pressure capacity

\begin{tabular}{lcccc}
\hline Sealing gaps/mm & 0.4 & 0.5 & 0.6 & 0.7 \\
\hline $\begin{array}{l}\text { Pressure capacities/MPa } \\
\begin{array}{l}\text { The pressure capacities after self-repai } \\
\text { ring/MPa }\end{array}\end{array}$ & 0.45 & 0.33 & 0.24 & 0.23 \\
\hline
\end{tabular}

For the leakage detection process, we can use the smoking gun to detect the leakage. The smoking gun was connected to the equipment, and the sealing chamber was filled with a certain proportion of helium-nitrogen gas mixture. When it reached the maximum pressure capacities, we can get the leakage values, the data was shown as Table 2 .

Tab.2 The leak rates of different sealing gaps

\begin{tabular}{lcccc}
\hline Sealing gaps $/ \mathrm{mm}$ & 0.4 & 0.5 & 0.6 & 0.7 \\
\hline vacuum $/ \mathrm{Pa}$ & 3 & 5 & 2 & 2 \\
Leakage rate $/ 10^{-8} \mathrm{~Pa} \mathrm{~m} \cdot \mathrm{s}^{-1}$ & 4.3 & 3.3 & 1.9 & 1.0 \\
\hline
\end{tabular}

\section{Analysis of the experiment data}

We can get the curve A from the data in the Table 1, as figure 4 shows. For a typical gap $0.2 \mathrm{~mm}$, single-stage sealing pressure capacity can reach at about $22 \mathrm{KPa}^{[7]}$, and for the magnetic fluid sealing structure designed in this paper, when the sealing gap is $0.4 \mathrm{~mm}$, the single-stage pressure capacity can reach at about $37 \mathrm{KPa}$. The pressure capacity decreases gradually with the sealing gap increases. And the single-stage pressure could reach $18 \mathrm{KPa}$ when the gap is $0.7 \mathrm{~mm}$. In addition, the magnetic fluid sealing has self-repair function, when the magnetic fluid is broken by high pressure, it can also maintain a good pressure capability. 


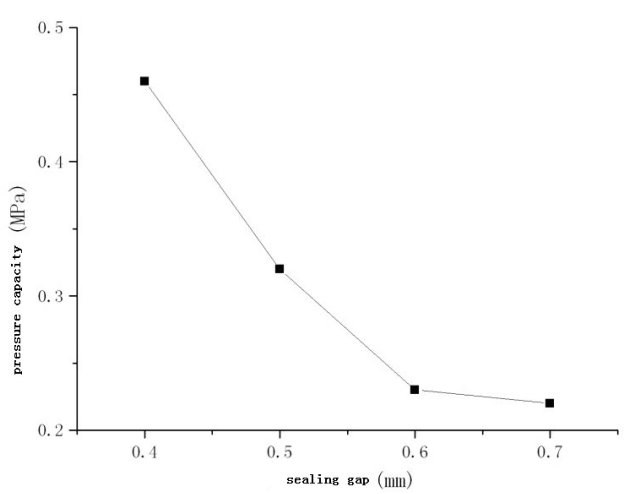

Fig.3 The relation between the sealing gaps and anti-pressure capacity Form Table 2 we can see that, with the sealing gap increases, the leakage rate which was measured has not increased, but has been decreased with the helium leak detector vacuum improved. So the magnetic fluid sealing can also be seen as "zero" leakage.

\section{Summary}

(1) The pressure capacity of magnetic fluid sealing decreases with the sealing gap increases, and the sealing pressure capacity has improved much greater than common structure.

(2) The magnetic fluid sealing has the self-repair functions, it could still have a good pressure capacity after restoring.

(3) In the present experimental conditions, the magnetic fluid "zero" leakage conclusion is still valid.

\section{References}

[1] LIU Tong-gang,YANG Zhi-yi: A New Design of Magnetic Fluid Seal for Liquids, 2005, Vol.15 No.4,366-369.

[2]SUN Mingli, LI Decai, CUI Hairong, et al:Journal of Beijing Jiaotong University,32(1),2008,p.116.

[3]Zhao Meng,Zou Jinbin,Hu Jianhui.An analysis on the magnetic fluid seal capacity. Journal of magnetism and magnetic materials.303(2006):428-431.

[4] D.Jamon,F.Donatini,A. Siblini, F.Royer, R.Perzynski. Experimental investigation on the magneto-optic effects of ferrofluids via dynamic measurements, Journal of magnetism and magnetic materials,321(2009)1148-1154.

[5] Li Decai, in: Theories and Applications of Magnetic liquid, edtied by Science Press, Beijing, 2003.

[6] P.V. Melenev,V.V.Rusakov,Yu.L.Raikher,R.Perzyski: Ground magnetic state of an assembly of single-domain particles confined in a spherical layer, Journal of magnetism and magnetic materials,321(2009)663-666.

[7]Feifei Xing, Decai Li, Xiaolong Yang. Experimental Study of Large Gap Magnetic Fluid Static Sealing. Key Engineering Materials.492(2012):283-286. 